Check for updates

Cite this: RSC Adv., 2018, 8, 1371

\title{
A surfactant-free microemulsion composed of isopentyl acetate, $n$-propanol, and water $\dagger$
}

\author{
Yuan Liu, ${ }^{a}$ Jie Xu, ${ }^{\star a}$ Huanhuan Deng, ${ }^{a}$ Jiaxin Song ${ }^{a}$ and Wanguo Hou (D) *b
}

It has been demonstrated that in the absence of traditional surfactants, microemulsions can form from a ternary mixture of oil, water, and an amphi-solvent. These microemulsions are called surfactant-free microemulsions (SFMES). To date, only a small number of SFME systems have been reported, and the current understanding of SFMEs is very limited. Herein, we report an SFME consisting of isopentyl acetate (IA), n-propanol, and water, in which IA (a simple ester compound) and $n$-propanol are used as the oil phase and amphi-solvent, respectively. The microstructures and structural transition of the SFME were investigated by cyclic voltammetry, fluorescence spectroscopy, and UV-visible spectroscopy techniques. Moreover, three kinds of microstructures, namely, oil-in-water $(\mathrm{O} / \mathrm{W})$, bicontinuous $(\mathrm{BC})$, and water-inoil (W/O), have been identified in the SFME, which are directly verified by cryo-TEM observations. A change in the composition of the SFME may lead to a structural transition from O/W through BC to W/O or vice versa, which is similar to the case of traditional surfactant-based microemulsions (SBMEs). To the best of our knowledge, this is the first time that the microstructures and structural transition of an SFME obtained using a simple ester compound as the oil phase have been identified.

Received 20th November 2017 Accepted 19th December 2017

DOI: 10.1039/c7ra12594a

rsc.li/rsc-advances water. Subsequently, the formation of SFMEs was confirmed in some surfactant-free ternary systems. ${ }^{5-21}$ The structures, properties, and formation mechanism of SFMEs have been investigated. ${ }^{1,222-24}$ It has been preliminarily demonstrated that the structures and properties of SFMEs are similar to those of traditional SBMEs to some extent. ${ }^{2}$ For instance, similar to SBMEs, ${ }^{3}$ SFMEs can exhibit three structures: oil-inwater $(\mathrm{O} / \mathrm{W})$, bicontinuous $(\mathrm{BC})$, and water-in-oil (W/O) structures, and the three kinds of structures can be translated into each other with change in the composition of the system. ${ }^{7,-11,13-15,19,21}$ In addition, similar to SBMEs ${ }^{, 5}$ SFMEs are expected to have extensive potential applications ${ }^{2}$ such as in enzymatic reactions, ${ }^{26}$ chemical reactions, ${ }^{27-29}$ and nanoparticle synthesis. ${ }^{30-32}$ In particular, SFMEs when used as reaction media may avoid some problems, such as complex separation and purification procedures, possible ecotoxicity, and high material cost, caused by the existence of large amounts of surfactants ( $>10 \mathrm{wt} \%)$ in SBMEs. ${ }^{2}$ However, SFMEs have received very less attention as compared to SBMEs. ${ }^{22}$ Over the past few decades, more than 10000 studies dealing with SBMEs have been reported in the literature, ${ }^{12}$ whereas less than 60 studies dealing with SBMEs have been reported. ${ }^{2}$ In addition, less than 20 SFME systems have been reported to date. ${ }^{2}$ Consequently, the current understanding of SFMEs is still very limited, and many issues about SFMEs need to be studied; one of these issues is to establish whether SFME formation is a general phenomenon. It is desirable to find more SFME systems, which can provide information for understanding the nature of SFMEs.
${ }^{a}$ State Key Laboratory Base of Eco-chemical Engineering, Qingdao University of Science and Technology, 266042, Qingdao, P. R. China. E-mail: xujie@qust.edu.cn; Fax: +86531-88564750; Tel: +86-531-88365460

${ }^{b}$ Key Laboratory for Colloid and Interface Chemistry (Ministry of Education), Shandong University, 250100, Jinan, P. R. China. E-mail: wghou@sdu.edu.cn

$\dagger$ Electronic supplementary information (ESI) available. See DOI: 10.1039/c7ra12594a 
Herein, we report an SFME consisting of isopentyl acetate (IA), $n$-propanol, and water, in which IA and $n$-propanol are used as the oil phase and amphi-solvent, respectively. The structures and structural transition of the SFME were investigated using cyclic voltammetry, fluorescence spectroscopy, and UV-visible spectroscopy. Similar to the traditional SBMEs, the SFME exhibits $\mathrm{W} / \mathrm{O}, \mathrm{BC}$, and $\mathrm{O} / \mathrm{W}$ structures depending on the composition of the ternary system. The three kinds of structures were confirmed by cryogenic transmission electron microscopy (cryo-TEM) observations. The Kunz group ${ }^{\mathbf{1 6 , 2 0}}$ has investigated the phase behavior of ester-compound-containing ternary systems including geranyl acetate/ethanol/water ${ }^{16}$ and diethyl adipate/tetrahydrofurfuryl alcohol/deep eutectic solvent (ethylene glycol-choline chloride or urea-choline chloride) ${ }^{20}$ and revealed the formation of SFMEs in these systems by dynamic/static light scattering and small-angle X-ray scattering measurements. However, the microstructures of the SFMEs were not identified. To the best of our knowledge, this is the first study in which the microstructures and structural transition of an SFME obtained using a simple ester compound as the oil phase have been identified. This study provides a better understanding of SFME construction. In addition, the SFME may have some specific applications, such as in material preparation, reaction engineering, and separation, due to its surfactant-free nature.

\section{Experimental}

\subsection{Chemicals}

All the chemicals used in this study were of analytical reagent grade. Isopentyl acetate (IA, purity $\geq 99.5 \%$ ) and $n$-propanol (purity $\geq 99.8 \%$ ) were purchased from Sinopharm Chemical Reagent Co., Ltd., China, and their molecular structures are shown in Fig. S1 in the ESI. $\dagger$ Pyrene (GC grade) was purchased from Sigma-Aldrich, USA. Methyl orange (MO) and potassium ferricyanide $\left(\mathrm{K}_{3} \mathrm{Fe}(\mathrm{CN})_{6}\right)$ were purchased from Tianjin Chemical Reagents Co., China. IA was purified before use by washing with a cold saturated $\mathrm{NaCl} / \mathrm{NaOH}$ solution $(\mathrm{pH}=10-11)$, drying with 4A molecular sieve, and a final distilling process to remove its free acid impurity. Other chemicals were used as received. Ultrapure water with a resistivity of $18.3 \mathrm{M} \Omega \mathrm{cm}$ was obtained using an AFZ-1000-U purification system (Chongqing Ever Young Enterprises Development Co. Ltd., China).

\subsection{Phase diagram construction}

The phase diagram of the ternary system IA/ $n$-propanol/water was constructed at $25.0 \pm 0.2{ }^{\circ} \mathrm{C}$ by titration with $n$-propanol for IA/water mixtures. An IA/water mixture with a desired volume ratio $\left(R_{\mathrm{IA} / \mathrm{W}}\right)$ was prepared in a dry test-tube. An appropriate volume of $n$-propanol was added to the mixture under magnetic stirring. The phase boundary was determined by observing the transition from turbidity to transparency or vice versa. Repetition of this experiment for other $R_{\mathrm{IA} / \mathrm{W}}$ values allowed the phase diagram to be established. The entire procedure was repeated three times, and an average value was used. The component content was expressed as the volume fraction in the ternary phase diagram.

\subsection{Cyclic voltammetry measurements}

Cyclic voltammetry measurements were performed using a CHI model 660D electrochemical workstation (Shanghai Chenhua Instrument Factory, China) with a three-electrode cell, which consisted of a glass-carbon working electrode (electrode area $0.07 \mathrm{~cm}^{2}$ ), a $\mathrm{AgCl} / \mathrm{Ag}$ reference electrode, and a Pt wire counter electrode. The space between adjacent electrodes was $2.0 \mathrm{~cm}$. Before each measurement, the working electrode was polished using a $0.05 \mathrm{~mm}$ aluminum oxide slurry and then washed carefully with ultrapure water. The electrode was then ultrasonicated in ultrapure water for approximately $5 \mathrm{~min}$ before use. The potential was scanned between $-0.2 \mathrm{~V}$ and $0.8 \mathrm{~V}$, with a sweep rate range of $20-100 \mathrm{mV} \mathrm{s}^{-1}$. All experiments were carried out at $25.0 \pm 0.2{ }^{\circ} \mathrm{C}$ under a nitrogen atmosphere to avoid oxygen effects. $\mathrm{K}_{3} \mathrm{Fe}(\mathrm{CN})_{6}$ was selected as the electroactive probe, with a fixed concentration of $0.65 \mathrm{~g} \mathrm{~L}^{-1}$.

Based on the peak current data obtained at various sweep rates, the diffusion coefficient of the electroactive probe $D_{\mathrm{p}}$ $\left(\mathrm{m}^{2} \mathrm{~s}^{-1}\right)$ in microemulsions can be estimated from the RandlesSevcik equation: ${ }^{33}$

$$
i_{\mathrm{p}}=0.477 A c\left(\frac{F^{3} n_{\mathrm{e}}{ }^{3} D_{\mathrm{p}} v}{R T}\right)^{1 / 2}
$$

where $i_{\mathrm{p}}$ is the peak current for a redox-active reversible system, $A$ is the area of the working electrode, $c$ is the concentration of the electroactive probe, $R$ is the gas constant, $T$ is the absolute temperature, $F$ is the Faraday constant, $n_{\mathrm{e}}$ is the number of electrons involved in oxidation or reduction, and $v$ is the sweep rate. The $D_{\mathrm{p}}$ of the electroactive probe can be calculated from the slope of an $i_{\mathrm{p}}-v^{1 / 2}$ linear plot.

\subsection{Fluorescence measurements}

Fluorescence emission spectra were obtained using a spectrofluorometer (F-2700, Hitachi, Japan) with a $1 \mathrm{~cm}$ path-length quartz cell using pyrene as a fluorescent probe at $25.0 \pm$ $0.2{ }^{\circ} \mathrm{C}$. The pyrene solution in ethanol was placed in a dry testtube, and ethanol was evaporated by placing the test-tube in a water bath. Appropriate amounts of IA, $n$-propanol, and water were then added to the test-tube, and the pyrene concentration in the mixture was maintained at $4.95 \times 10^{-5} \mathrm{~mol} \mathrm{~L}^{-1}$. The excitation and emission slits were set at a $0.5 \mathrm{~nm}$ band-pass. The fluorescence emission spectrum of pyrene from 350 to $420 \mathrm{~nm}$ was obtained after excitation at $334 \mathrm{~nm}$. The fluorescence emission spectrum of each sample was obtained after allowing sufficient time $(\sim 48 \mathrm{~h})$ for equilibration.

\subsection{UV-visible adsorption measurements}

UV-visible spectra were obtained using a computer-controlled UV-visible spectrometer (TU-1901, Beijing Purkinje General Instrument Co., Ltd., China), with a $1 \mathrm{~cm}$ path-length quartz cell using MO as a probe. Appropriate amounts of IA, $n$-propanol, and water containing a desired amount of MO were uniformly 
mixed in advance, and the resultant sample containing $5.0 \mathrm{mg} \mathrm{L}{ }^{-1} \mathrm{MO}$ was left for $48 \mathrm{~h}$ prior to measurements. The spectrum scanning was carried out at $25.0 \pm 0.2{ }^{\circ} \mathrm{C}$ with $0.2 \mathrm{~nm}$ resolution.

\subsection{Dynamic light scattering measurements}

Dynamic light scattering (DLS) measurements were performed at $25.0 \pm 0.2{ }^{\circ} \mathrm{C}$ using a Nano ZS90 instrument (Malvern, England) with a $4 \mathrm{~mW}$ He-Ne laser $(\lambda=632.8 \mathrm{~nm})$. Before measurements, the samples were filtered through a polycarbonate membrane $(0.45 \mu \mathrm{m}$ pore size) to remove any dust particles. All the scattering photons were obtained at a $90^{\circ}$ scattering angle. The scattering data were analyzed using the CONTIN method to obtain the size distribution and average hydrodynamic diameter $\left(d_{\mathrm{h}}\right)$ of the test samples. The viscosity and refractive index measurements were carried out using a rotational rheometer (RheolabQC, Anton-Paar, Austria) and an Abbe refractometer (WAY-2S, Shanghai Instrument Physical Optics Instrument Co., Ltd., China), respectively, at $25.0 \pm$ $0.2{ }^{\circ} \mathrm{C}$.

\subsection{Cryo-TEM observations}

Cryogenic transmission electron microscopy (cryo-TEM) observations were performed to determine the structures of microemulsions. The cryo-TEM samples were prepared in a controlled-environment vitrification system (Cryoplunge TM3, USA) at $25{ }^{\circ} \mathrm{C}$ and $95 \%$ relative humidity. A $4 \mathrm{~mL}$ aliquot of the sample was loaded onto a carbon-coated copper grid. The excess solution was then blotted off with filter paper, and a thin film suspended on the mesh holes was produced. After about $5 \mathrm{~s}$, the sample-loaded grid was quickly put in liquid ethane (cooled by liquid nitrogen). The vitrified sample was transferred into a cryogenic specimen holder (Gatan 626) and observed using a JEM-1400 TEM (JEOL, Japan) at approximately $-174{ }^{\circ} \mathrm{C}$ with an accelerating voltage of $120 \mathrm{kV}$. The images were obtained using a Gatan multiscan CCD and processed with a Digital micrograph. All samples used for cryo-TEM were filtered through a $0.45 \mu \mathrm{m}$ filter prior to measurements.

\section{Results and discussion}

\subsection{Phase behavior of the IA/n-propanol/water ternary system}

Fig. 1 shows the ternary phase diagram of the system IA $/ n$ propanol/water at $25.0 \pm 0.2{ }^{\circ} \mathrm{C}$, in which the component content in the system is expressed as the volume fraction $\left(f_{\mathrm{i}}\right.$, where i represents IA, $n$-propanol or water). Moreover, two regions are observed in the diagram: one is a single-phase region (blank region), and the other is a multiphase region (shadow region). In the single-phase region, the ternary systems are optically isotropic and transparent, whereas in the multiphase region, they are turbid under stirring and break quickly into two phases when left to stand. The single-phase region occupies about $55 \%$ area in the total phase diagram. This diagram indicates that immiscible IA and water can stably coexist in a homogeneous system with the aid of a certain

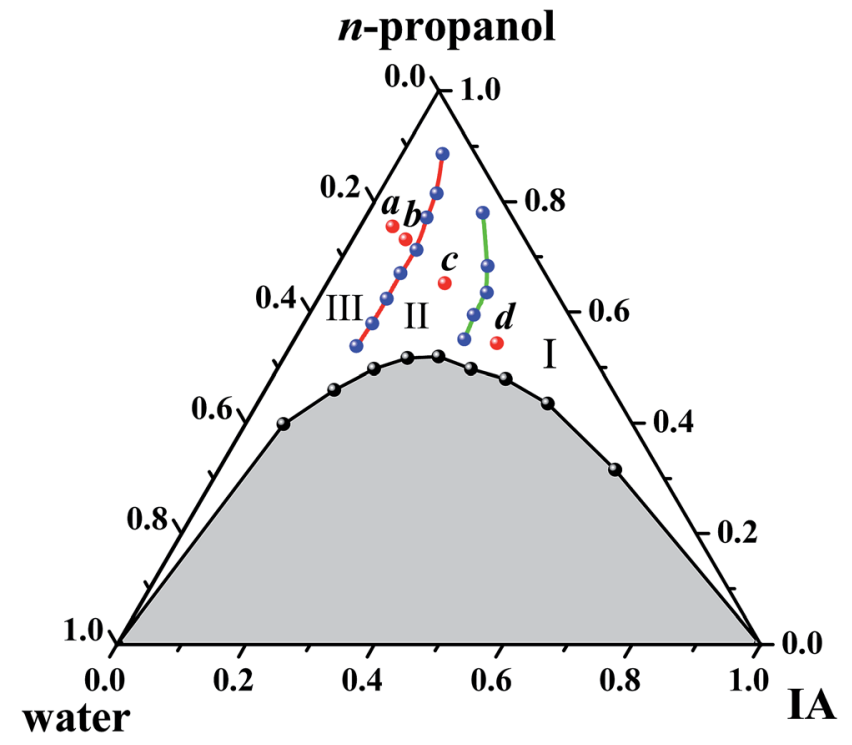

Fig. 1 Phase diagram of the $\mid \mathrm{A} / \mathrm{n}$-propanol/water system at $25 \pm$ $0.2{ }^{\circ} \mathrm{C}$. I, II, and III represent $\mathrm{W} / \mathrm{O}, \mathrm{BC}$, and $\mathrm{O} / \mathrm{W}$ subregions, respectively. Samples $a, b, c$, and $d$ were chosen for cryo-TEM observations.

amount of $n$-propanol. Based on a previous study, ${ }^{2}$ SFMEs most likely form in the single-phase region. In addition, the singlephase channel extending from oil-rich to water-rich regions obtained herein is suitable for studying the structural transition.

\subsection{Structures and structural transition of microemulsions}

Cyclic voltammetry. Cyclic voltammetry measurements were performed for the ternary mixtures in the single-phase region using $\mathrm{K}_{3} \mathrm{Fe}(\mathrm{CN})_{6}$ as the electroactive probe to determine the microstructures and structural transition. In the measurements, $20 \mathrm{mM} \mathrm{KCl}$ was added in the water phase to improve the conductivity of systems. This low concentration of $\mathrm{KCl}$ cannot obviously affect the microstructures of SFMEs. ${ }^{1,22}$ Because only the diffusion-controlled electrochemical charge transfer is suitable for studying the microstructure of microemulsions, ${ }^{34}$ the change in $i_{\mathrm{p}}$ with $v$ has been first determined for two mixtures, in which the volume fraction of IA $\left(f_{\mathrm{IA}}\right)$ is 0.05 and the volume ratios of $n$-propanol to water $\left(R_{\mathrm{P} / \mathrm{W}}\right)$ are 8.0/2.0 and 7.0/3.0. The $i_{\mathrm{p}}-v^{1 / 2}$ plots obtained for the two mixtures are all straight lines passing through the origin (Fig. S2, ESI $\dagger$ ), indicating that the electron transport of the electrode reaction in the mixtures is diffusion-controlled. ${ }^{35,36}$ Therefore, $\mathrm{K}_{3} \mathrm{Fe}(\mathrm{CN})_{6}$ is a suitable electroactive probe for studying the microstructures and structural transition of the microemulsions.

Along various IA dilution lines with different $R_{\mathrm{P} / \mathrm{W}}$ values (Fig. S3, ESI $\dagger$ ), the change in $D_{\mathrm{p}}$ of $\mathrm{K}_{3} \mathrm{Fe}(\mathrm{CN})_{6}$ in the ternary system over the single-phase region was examined. A typical plot of $D_{\mathrm{p}}$ versus $f_{\mathrm{IA}}$ for $R_{\mathrm{P} / \mathrm{W}}=8.0 / 2.0$ is shown in Fig. 2 . The $D_{\mathrm{p}}$ decreases monotonically with the increasing $f_{\text {IA }}$ over the entire single-phase region. However, two breakpoints are observed in the $D_{\mathrm{p}}-f_{\mathrm{IA}}$ plot. The whole curve can therefore be divided into 


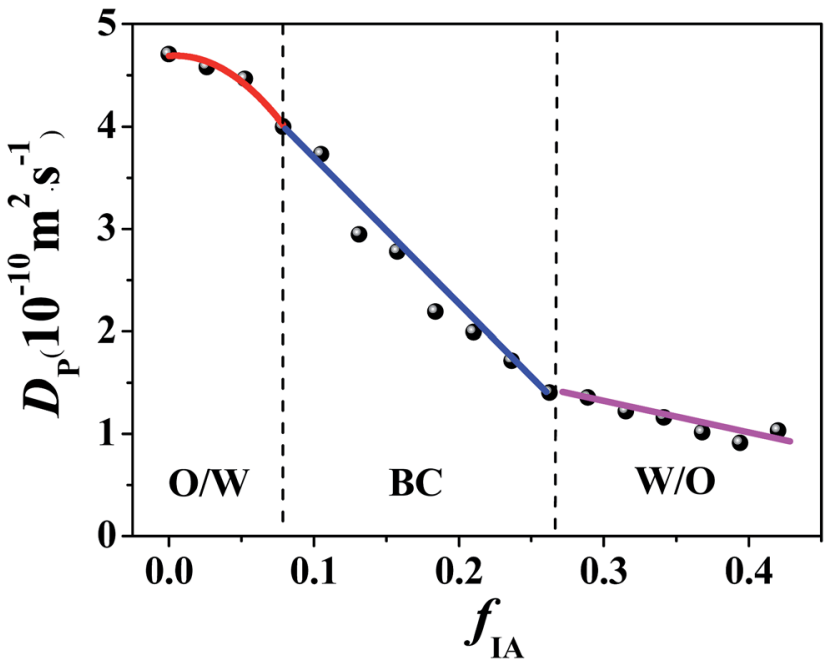

Fig. 2 Diffusion coefficient of $\mathrm{K}_{3} \mathrm{Fe}(\mathrm{CN})_{6}$ in the $\mathrm{IA} / n$-propanol/water ternary system with $R_{\mathrm{P} / \mathrm{W}}=8.0 / 2.0$ as a function of $f_{\mathrm{IA}}$. The $\mathrm{K}_{3} \mathrm{Fe}(\mathrm{CN})_{6}$ concentration is $0.65 \mathrm{~g} \mathrm{~L}^{-1}$.

three successive stages: an initial slow decrease, a subsequent quicker decrease, and a final slower decrease, as indicated in Fig. 2. Similar results are obtained for $R_{\mathrm{B} / \mathrm{P}}=9.5 / 0.5,9.0 / 1.0,8.5 /$ 1.5, and 7.5/2.5 (Fig. S4, ESI $\dagger$ ). Based on previous studies, ${ }^{\mathbf{1 4}, 15,33,37,38}$ the discontinuous change in $D_{\mathrm{p}}$ can be attributed to the change in the microstructure of the $\mathrm{K}_{3} \mathrm{Fe}(\mathrm{CN})_{6}{ }^{-}$ located environment, i.e., a microemulsion forms in the ternary system and its microstructure is changed with a change in $f_{\mathrm{IA}}$. Generally, when an electroactive probe is completely solubilized in dispersed droplets, a lower $D_{\mathrm{p}}$ value will be obtained in comparison with that from a continuous phase. This is because the probe located in droplets diffuses with the droplets, and the obtained $D_{\mathrm{p}}$ value corresponds to the apparent diffusion coefficient of the droplets. ${ }^{37,38}$ In this study, the electroactive probe $\mathrm{K}_{3} \mathrm{Fe}(\mathrm{CN})_{6}$ is expected to preferentially probe the water environment because of its limited solubility in IA. Therefore, the high $D_{\mathrm{p}}$ value at low $f_{\mathrm{IA}}\left(e . g .,<0.08\right.$ for $\left.R_{\mathrm{P} / \mathrm{W}}=8.0 / 2.0\right)$ suggests the formation of an $\mathrm{O} / \mathrm{W}$ type microemulsion, which corresponds to the diffusion of the probe in the water-rich continuous phase. The low $D_{\mathrm{p}}$ value at high $f_{\mathrm{IA}}\left(e . g\right.$.,$f_{\mathrm{IA}}>0.27$ for $R_{\mathrm{P} / \mathrm{W}}$ $=8.0 / 2.0$ ) suggests the formation of a W/O type microemulsion, which corresponds to the diffusion of the water-rich droplets. At intermediate $f_{\mathrm{IA}}\left(e . g\right.$., $0.08<f_{\mathrm{IA}}<0.27$ for $\left.R_{\mathrm{P} / \mathrm{W}}=8.0 / 2.0\right)$, the change rate in $D_{\mathrm{p}}$ with $f_{\mathrm{IA}}$ is different from those at both high and low $f_{\mathrm{IA}}$; this suggests the formation of a BC microstructure. ${ }^{\mathbf{1 4 , 1 5 , 3 3 , 3 5 , 3 7 , 3 9 , 4 0}}$

The cyclic voltammetry data demonstrate that with the increasing $f_{\mathrm{IA}}$, a structural transition of the microemulsion occurs, i.e., from $\mathrm{O} / \mathrm{W}$ through $\mathrm{BC}$ to $\mathrm{W} / \mathrm{O}$. Notably, only one breakpoint appears for the ternary systems with $R_{\mathrm{P} / \mathrm{W}}=7.0 / 3.0$, 6.5.0/3.5, and 6.0/4.0 (Fig. S4, ESI $\dagger$ ), suggesting that the transition from $\mathrm{W} / \mathrm{O}$ to $\mathrm{BC}$ occurs along the IA dilution lines. Furthermore, no obvious breakpoint appears for the ternary system with $R_{\mathrm{P} / \mathrm{W}}=5.5 / 4.5$ (Fig. S4, ESI $\dagger$ ); this suggests that no phase transition occurs along the IA dilution line. ${ }^{\mathbf{1 4 2 1}}$
Fluorescence spectroscopy. Hydrophobic pyrene is a widely used fluorescence probe, and its fluorescence emission spectrum shows five vibronic bands at 373, 378, 383, 388, and $393 \mathrm{~nm}$. The intensity ratio of the fifth peak to the first peak, $I_{393} / I_{373}$, is very sensitive to the polarity of pyrene-located microenvironments. A high $I_{393} / I_{373}$ value represents a low polarity of the microenvironment. ${ }^{\mathbf{4 1 , 4 2}}$ In our previous study, ${ }^{15,21}$ we have found that a discontinuous change in $I_{393} / I_{373}$ with $f_{\mathrm{w}}$ or $f_{\mathrm{o}}$ may occur as the microstructure of microemulsion changes. Therefore, fluorescence spectroscopy of pyrene can be used to identify the structural transition of microemulsions. Along various IA dilution lines with different $R_{\mathrm{P} / \mathrm{W}}$ values (9.0/1.0, 8.0/ 2.0, and 7.0/3.0), the change in $I_{393} / I_{373}$ of pyrene in the microemulsion was determined. Fig. 3 shows a typical curve of $I_{393} / I_{373}$ versus $f_{\mathrm{IA}}$ for $R_{\mathrm{P} / \mathrm{W}}=8.0 / 2.0$. The $I_{393} / I_{373}$ value decreases monotonically with the increasing $f_{\mathrm{IA}}$, and two breakpoints are clearly observed. Similar results are obtained for $R_{\mathrm{P} / \mathrm{W}}=9.0 / 1.0$ (Fig. S5C, ESI $\dagger$ ).

The $I_{393} / I_{373}$ values of pyrene in bulk water, IA, and $n$-propanol were determined to be $0.824,1.035$, and 1.075 , respectively, indicating that the order of the polarity for the three components is water $>$ IA $>n$-propanol. In addition, hydrophobic pyrene is expected to preferentially probe the IA microenvironment because of its limited solubility in water. Notably, the IA and water phases in the SFMEs studied herein actually consist of IA/n-propanol and water/n-propanol solutions, respectively, owing to the fact that $n$-propanol is miscible with both IA and water. With the increasing $f_{\mathrm{IA}}$, the $n$-propanol content in the IA/ $n$-propanol phase decreases; this may lead to a decrease in $I_{393} /$ $I_{373}$ owing to the $I_{393} / I_{373}$ value of $n$-propanol being higher than that of IA. In addition, the $f_{\mathrm{IA}}$ values corresponding to the breakpoints are very close to those observed in cyclic voltammetry measurements (Fig. S5, ESI $\dagger$ ). Therefore, the discontinuous change in $I_{393} / I_{373}$ observed herein can be attributed to the structural transition of the microemulsion with changing $f_{\mathrm{IA}}$. That is, the three changing stages in $I_{393} / I_{373}$ observed from low to high $f_{\mathrm{IA}}$ correspond to $\mathrm{W} / \mathrm{O}, \mathrm{BC}$, and $\mathrm{O} / \mathrm{W}$ microstructures.

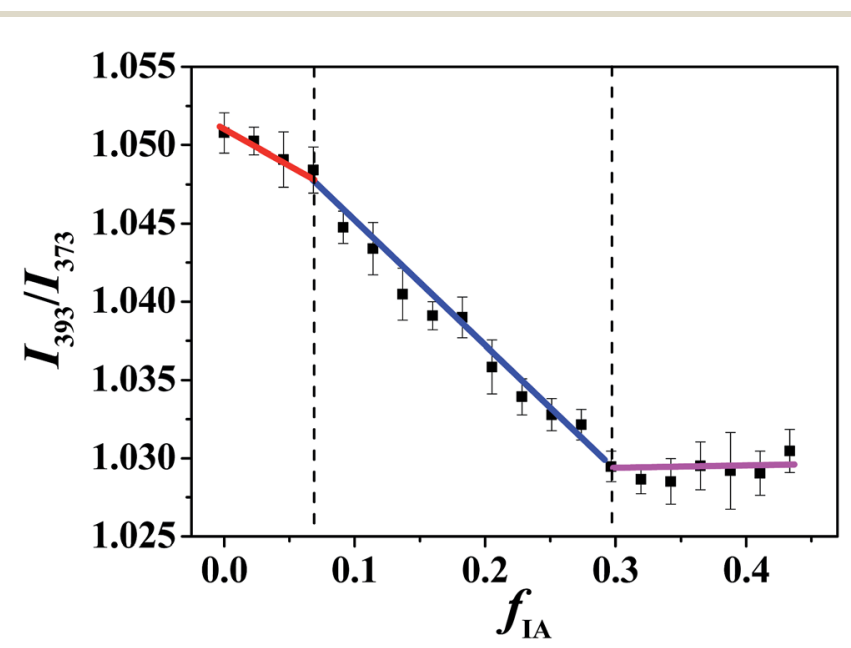

Fig. 3 Intensity ratio $/ 393 / / 373$ of pyrene in microemulsions at $R_{\mathrm{P} / \mathrm{W}}=$ $8.0 / 2.0$ as a function of $f_{\mathrm{IA}}$. The pyrene concentration is $4.95 \times$ $10^{-5} \mathrm{~mol} \mathrm{~L}^{-1}$. 
Notably, only one breakpoint appears for the ternary system with $R_{\mathrm{P} / \mathrm{W}}=7.0 / 3.0$ (Fig. S6B, ESI $\dagger$ ), suggesting that only a transition from $\mathrm{W} / \mathrm{O}$ to $\mathrm{BC}$ occurs along the IA dilution line, which is similar to the cyclic voltammetry result.

UV-visible spectroscopy. MO is a widely used dye probe, and its visible absorption maximum $\left(\lambda_{\max }\right)$ is sensitive to its local microenvironment. ${ }^{33,43}$ A high polarity of MO-located microenvironments may result in a high $\lambda_{\max }$ value. In our previous study, ${ }^{15,21}$ we have found that a discontinuous change in $\lambda_{\max }$ with $f_{\mathrm{w}}$ or $f_{\mathrm{o}}$ may occur as the microstructure of microemulsion changes. Therefore, UV-visible spectroscopy of MO can be used to identify the structural transition of microemulsions. Along various IA dilution lines with the given $R_{\mathrm{P} / \mathrm{W}}$ values (9.0/1.0, 8.0/ 2.0, and 7.0/3.0), the change in $\lambda_{\max }$ of MO in the microemulsions was determined. Fig. 4 shows a typical curve of $\lambda_{\max }$ versus $f_{\mathrm{IA}}$ for $R_{\mathrm{P} / \mathrm{W}}=8.0 / 2.0$. With the increasing $f_{\mathrm{IA}}$, the $\lambda_{\max }$ value initially decreases, then remains almost unchanged, and finally decreases again. Moreover, two breakpoints appear in the whole $\lambda_{\max }-f_{\mathrm{IA}}$ plot. A similar result is obtained for $R_{\mathrm{P} / \mathrm{W}}=$ 9.0/1.0 (Fig. S5E, ESI $\dagger$ ). MO is expected to preferentially probe the water microenvironment due to its good water-solubility. In addition, MO prefers to locate at the water/IA interfacial phase, ${ }^{15,21}$ thereby being also sensitive to the IA content. The $\lambda_{\max }$ value of MO in bulk water $(464.2 \mathrm{~nm})$ is obviously higher than that in bulk IA $(416.4 \mathrm{~nm})$. Therefore, the increase of $f_{\mathrm{IA}}$ leads to a decrease in $\lambda_{\max }$ of MO. The almost unchanged $\lambda_{\max }$ at intermediate $f_{\text {IA }}$ suggests that the corresponding microstructure is different from those formed at low and high $f_{\mathrm{IA}}$. Furthermore, the $f_{\mathrm{IA}}$ values corresponding to the breakpoints are very close to those observed in cyclic voltammetry and fluorescence spectroscopy measurements (Fig. S5, ESI $\dagger$ ). Therefore, it can be concluded that the three changing stages in $\lambda_{\max }$ observed from low to high $f_{\mathrm{IA}}$ correspond to the formation of $\mathrm{W} / \mathrm{O}, \mathrm{BC}$, and $\mathrm{O} /$ $\mathrm{W}$ microstructures. Similar to the cyclic voltammetry and fluorescence results, only one breakpoint is observed for $R_{\mathrm{P} / \mathrm{W}}=7.0$ / 3.0 (Fig. S6C, ESI†).

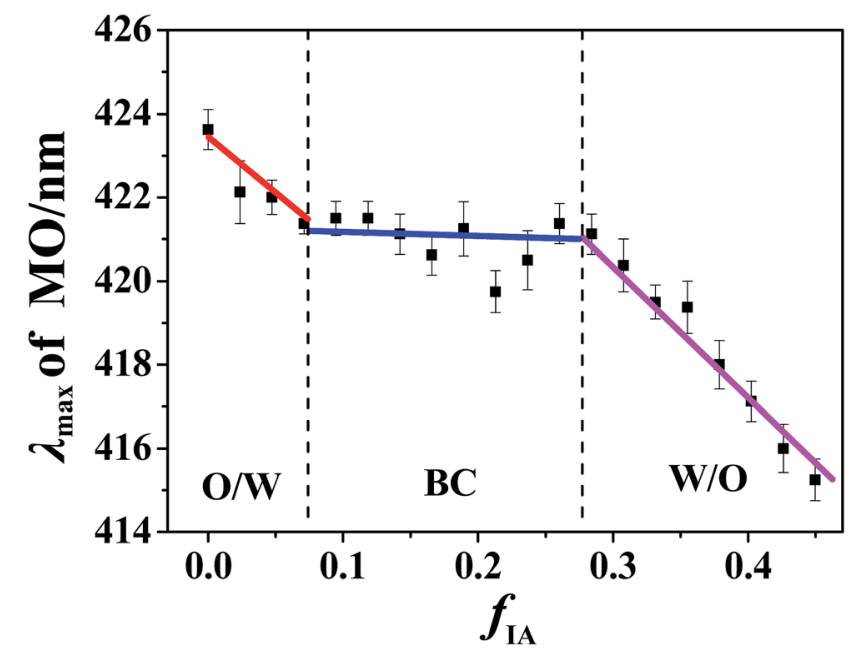

Fig. $4 \mathrm{MO} \lambda_{\max }$ as a function of $f_{\mathrm{IA}}$ in microemulsions at $R_{\mathrm{P} / \mathrm{W}}=8.0 /$ 2.0. The $\mathrm{MO}$ concentration in microemulsions is $5 \mathrm{mg} \mathrm{L}^{-1}$.
Subregions of single-phase microemulsion region. The abovementioned results of cyclic voltammetry and fluorescence and UV-visible spectroscopies demonstrate that the IA $/ n$ propanol/water ternary system can form microemulsions. With the increasing $f_{\mathrm{IA}}$, the microstructures of the microemulsions can translate from $\mathrm{W} / \mathrm{O}$ through $\mathrm{BC}$ to $\mathrm{O} / \mathrm{W}$. The boundaries for the three microstructures obtained by the three techniques are consistent with each other (Fig. S5 and S6, ESI $\dagger$ ). The three subregions corresponding to the $\mathrm{W} / \mathrm{O}, \mathrm{BC}$, and $\mathrm{O} / \mathrm{W}$ microemulsions, respectively, are marked in Fig. 1. The uncertainty in locating the boundaries of different subregions is estimated to be less than $10 \%$. The microstructures and structural transition observed herein are similar to those reported for other SFMEs ${ }^{2,7,9,13-15,19,21}$ and SBMEs. ${ }^{33,35,38-40}$

\subsection{Cryo-TEM and DLS studies}

Along the IA dilution line with $R_{\mathrm{P} / \mathrm{W}}=8.0 / 2.0$, four samples with $f_{\mathrm{IA}}=0.050,0.083,0.183$, and 0.318 (denoted as $a, b, c$, and $d$, respectively) were chosen for cryo-TEM observations. The samples $a$ and $b$ fall in the $\mathrm{O} / \mathrm{W}$ subregion, and the samples $c$ and $d$ fall in the $\mathrm{BC}$ and $\mathrm{W} / \mathrm{O}$ subregions, respectively, as marked in Fig. 1. The cryo-TEM images of the four samples are shown in Fig. 5 (and Fig. S7, ESI $\dagger$ ). Spherical droplets are observed for the samples $a, b$, and $d$, demonstrating the existence of discrete droplets in the systems. The average diameters of droplets for the samples $a, b$, and $d$ are measured to be $\sim 80$, 200 , and $120 \mathrm{~nm}$, respectively. For the sample $c$, a network structure (or a sponge-like structure) formed by the interconnection of droplets is observed, corresponding to both IA and water being continuous phases in the microstructure. The cryoTEM results directly verify the formation of $\mathrm{W} / \mathrm{O}, \mathrm{BC}$, and $\mathrm{O} / \mathrm{W}$ microemulsions.

To further determine the sizes of the dispersed droplets in the W/O and O/W SFMEs, DLS measurements were carried out for the samples $a$ and $b$ in the $\mathrm{O} / \mathrm{W}$ subregion and the sample $d$ in the W/O subregion (as marked in Fig. 1), as shown in Fig. 6. The $d_{\mathrm{h}}$ values of the samples $a, b$, and $d$ are 77, 198, and $167 \mathrm{~nm}$, respectively, close to their sizes observed by cryo-TEM.

\subsection{Stability of microemulsions}

The stability of the microemulsions was examined by the following processes: (1) heat-storage at $80{ }^{\circ} \mathrm{C}$ for one week; (2) cold-storage at $-10{ }^{\circ} \mathrm{C}$ for one week; (3) freeze $\left(-10^{\circ} \mathrm{C}\right)$-thaw $\left(40{ }^{\circ} \mathrm{C}\right)$ cycle 3 times; and (4) centrifugation under $100 \mathrm{~g}$ for $15 \mathrm{~min}$. No changes in the appearance of the microemulsion systems are found after different treatments, which is consistent with the thermodynamic stability characteristics of microemulsions.

Notably, the mechanism of the SFME formation is still unclear. The decrease in the isopentyl acetate (IA)/water interfacial tension caused by the addition of $n$-propanol may play a key role in the formation of the SFME. Most likely, $n$-propanol is preferentially located in the interphase between IA and water, as suggested in the previous reports. ${ }^{\mathbf{5} 20}$ Many essential questions concerning the nature of SFMEs remain to be addressed. 

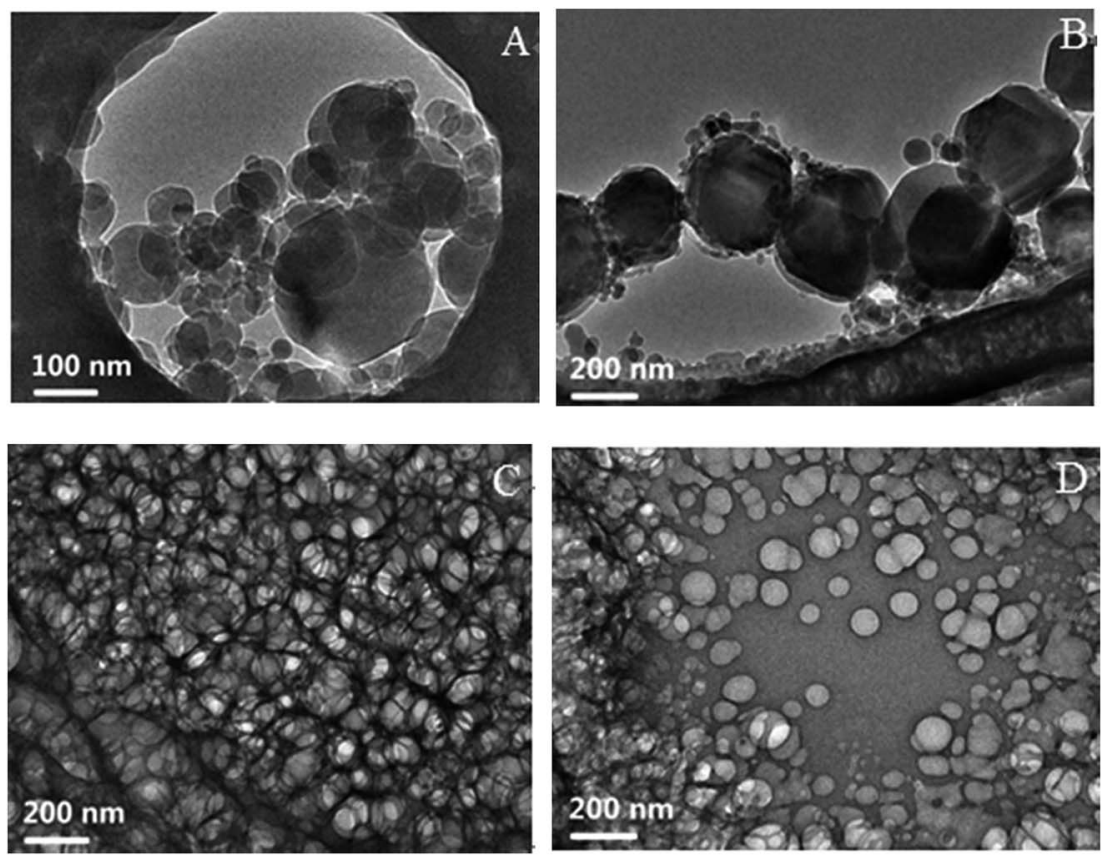

Fig. 5 Cryo-TEM images of the samples (A) $a,(B) b,(C) c$, and (D) $d$. The samples $a$ and $b$ fall in the O/W subregion, and the samples $c$ and $d$ fall in the $\mathrm{BC}$ and $\mathrm{W} / \mathrm{O}$ subregions, respectively, as marked in Fig. 1.

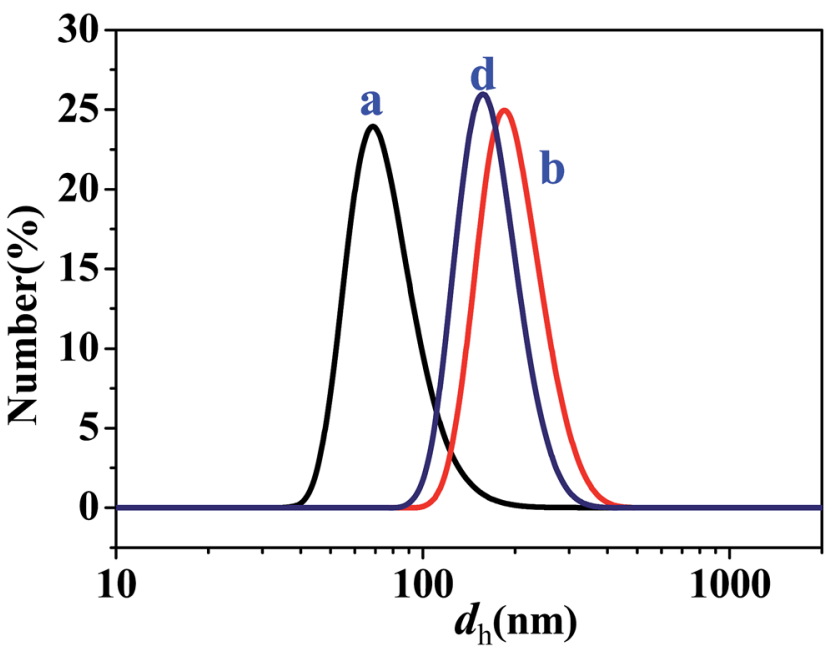

Fig. 6 Size distributions of dispersed droplets for the samples $a, b$, and $d$ as marked in Fig. 1.

\section{Conclusion}

The phase diagram of the ternary mixture of IA (oil phase), $n$ propanol (amphi-solvent), and water shows a single-phase and a multiphase region. Microemulsions, i.e., SFMEs, form in the single-phase region. The SFMEs may exhibit $\mathrm{O} / \mathrm{W}, \mathrm{BC}$, and $\mathrm{W} / \mathrm{O}$ microstructures, which are directly confirmed by cryo-TEM observations. A change in the composition of the SFMEs may lead to a structural transition from $\mathrm{O} / \mathrm{W}$ through $\mathrm{BC}$ to $\mathrm{W} / \mathrm{O}$ or vice versa, which is similar to the case of traditional SBMEs. This study provides evidence for the speculation that SFME formation may be a general phenomenon. These SFMEs may have specific applications in material preparation, reaction engineering, and separation because of their surfactant-free nature.

\section{Conflicts of interest}

The authors declare no competing financial interest.

\section{Acknowledgements}

This work was supported by the National Natural Science Foundation of China (No. 21403121 and 21573133) and the foundation of Key Laboratory of Colloid and Interface Chemistry (Shandong University), Ministry of Education, China (No. 201405).

\section{References}

1 S. Schöttl and D. Horinek, Curr. Opin. Colloid Interface Sci., 2016, 22, 8-13.

2 W. Hou and J. Xu, Curr. Opin. Colloid Interface Sci., 2016, 25, 67-74.

3 D. J. McClements, Soft Matter, 2012, 8, 1719-1729.

4 G. D. Smith, C. E. Donelan and R. E. Barden, J. Colloid Interface Sci., 1977, 60, 488-496.

5 G. Lund and S. L. Holt, J. Am. Oil Chem. Soc., 1980, 57, 264267.

6 J. Lara, G. Perron and J. E. Desnoyers, J. Phys. Chem., 1981, 85, 1600-1605.

7 Y. L. Khmelnitsky, A. V. Hoek, C. Veeger and A. J. W. G. Visser, J. Phys. Chem., 1989, 93, 872-878.

8 Y. R. Wang, L. L. Duan, S. Q. Cheng, B. Chai and F. Han, J. Dispersion Sci. Technol., 2008, 29, 280-283. 
9 P. Ni and W. G. Hou, Chin. J. Chem., 2008, 26, 1335-1338. 10 P. Ni and W. G. Hou, Chin. J. Chem., 2008, 26, 1985-1990.

11 M. L. Klossek, D. Touraud, T. Zemb and W. Kunz, ChemPhysChem, 2012, 13, 4116-4119.

12 J. Xu, A. Yin, J. Zhao, D. Li and W. Hou, J. Phys. Chem. B, 2013, 117, 450-456.

13 J. Xu, L. Zhang, A. Yin, W. Hou and Y. Yang, Soft Matter, 2013, 9, 6497-6504.

14 J. Xu, L. Zhang, C. Li, T. Zhan and W. Hou, RSC Adv., 2013, 3, 21494-21500.

15 J. Marcus, M. L. Klossek, D. Touraud and W. Kunz, Flavour Fragrance J., 2013, 28, 294-299.

16 O. Diat, M. L. Klossek, D. Touraud, B. Deme, I. Grillo, W. Kunz and T. Zemb, J. Appl. Crystallogr., 2013, 46, 16651669.

17 R. F. Hankel, P. E. Rojas, M. Cano-Sarabia, S. Sala, J. Veciana, A. Braeuer and N. Ventosa, Chem. Commun., 2014, 50, 82158218.

18 P. Boškovicć, V. Sokol, T. Zemb, D. Touraud and W. Kunz, J. Phys. Chem. B, 2015, 119, 9933-9939.

19 V. Fischer, J. Marcus, D. Touraud, O. Diat and W. Kunz, J. Colloid Interface Sci., 2015, 453, 186-193.

20 J. Xu, H. Deng, Y. Fu, Y. Chen, J. Zhang and W. Hou, Soft Matter, 2017, 13, 2067-2074.

21 S. Schöttl, J. Marcus, O. Diat, D. Touraud, W. Kunz, T. Zemb and D. Horinek, Chem. Sci., 2014, 5, 2949-2954.

22 P. Guilbaud and T. Zemb, Curr. Opin. Colloid Interface Sci., 2015, 20, 71-77.

23 T. N. Zemb, M. Klossek, T. Lopian, J. Marcus, S. Schöettl, D. Horinek, S. F. Prevost, D. Touraud, O. Diat, S. Marčelja and W. Kunz, Proc. Natl. Acad. Sci. U. S. A., 2016, 113, 4260-4265.

24 A. Xenakis, M. Zoumpanioti and H. Stamatis, Curr. Opin. Colloid Interface Sci., 2016, 22, 41-45.
25 N. F. Borys, S. L. Holt and R. E. Barden, J. Colloid Interface Sci., 1979, 71, 526-532.

26 D. K. Lavallee, E. Huggins and S. Lee, Inorg. Chem., 1982, 21, 1552-1553.

27 B. A. Keiser and S. L. Holt, Inorg. Chem., 1982, 21, 2323-2327.

28 J. Xu, L. Zhang, D. Li, J. Zhao and W. Hou, Colloid Polym. Sci., 2013, 291, 2515-2521.

29 T. Zhan, Y. Zhang, Q. Yang, H. Deng, J. Xu and W. Hou, Chem. Eng. J., 2016, 302, 459-465.

30 J. Xu, H. Deng, J. Song, J. Zhao, L. Zhang and W. Hou, J. Colloid Interface Sci., 2017, 505, 816-823.

31 Y. Gao, S. Han, B. Han, G. Li, D. Shen, Z. Li, J. Du, W. Hou and G. Zhang, Langmuir, 2005, 21, 5681-5684.

32 S. P. Moulik and B. K. Paul, Adv. Colloid Interface Sci., 1998, 78, 99-195.

33 M.-J. Schwuger, K. Stickdorn and R. Schomaecker, Chem. Rev., 1995, 95, 849-864.

34 K. Chokshi, S. Qutubuddin and A. Hussam, J. Colloid Interface Sci., 1989, 129, 315-326.

35 Y. Gao, N. Li, L. Zheng, X. Zhao, S. Zhang, B. Han, W. Hou and G. Li, Green Chem., 2006, 8, 43-49.

36 A. B. Mandal and B. U. Nair, J. Phys. Chem., 1991, 95, 90089013.

37 P. Guéring and B. Lindman, Langmuir, 1985, 1, 464-468.

38 Y. Gao, S. Han, B. X. Han, G. Li, D. Shen, Z. Li, J. Du, W. Hou and G. Zhang, Langmuir, 2005, 21, 5681-5684.

39 C. Mo, Langmuir, 2002, 18, 4047-4053.

40 Y. Gao, S. Wang, L. Zheng, S. Han, X. Zhang, D. Lu, L. Yu, Y. Ji and G. Zhang, J. Colloid Interface Sci., 2006, 301, 612616.

41 P. Lianos, J. Phys. Chem., 1982, 86, 1935-1937.

42 R. Sripriya, K. M. Raja, G. Santhosh, M. Chandrasekaran and M. Noel, J. Colloid Interface Sci., 2007, 314, 712-717.

43 D. M. Zhu and Z. A. Schelly, Langmuir, 1992, 8, 48-50. 